\title{
LAS TRES CARAS DEL RACISMO EPISTÉMICO EN EDUCACIÓN SUPERIOR
}

The three faces of epistemic racism in Higher Education

\author{
LUBI JEHINS GRANADA ANGULO ${ }^{1}$ \\ Universidad Pedagógica Nacional (Colombia) \\ lubigra@yahoo.com
}

\section{RESUMEN}

El presente artículo expone uno de los hallazgos centrales de la investigación doctoral Experiencias educativas de mujeres negras afrodescendientes: Un estudio interseccional, en el que se retoma el racismo epistémico evidenciado en las experiencias educativas de seis mujeres negras afrodescendientes con estudios doctorales en Colombia. Este racismo se revela especialmente en los espacios de educación superior, a través de tres formas: el pensamiento eurocéntrico, que da prelación a los saberes europeos y desvirtúa las epistemes producidas desde otras cosmovisiones no occidentalizadas; la invisibilización en el currículo, que se manifiesta cuando en los distintos espacios de educación formal no se muestran o se desconocen las elaboraciones epistémicas diversas a los parámetros eurocéntricos; y la marginalización académica que es identificada mediante la infravaloración de contenidos, la infravaloración de los grupos racializados y de la infrarrepresentación de mujeres y hombres negros afrodescendientes en los espacios de producción académica. Como ideas de

1 Doctora en educación del Doctorado Interinstitucional en Educación (DIE)- Sede Universidad Pedagógica Nacional (UPN). Magíster en Educación Universidad Pedagógica Nacional. Licenciada en Psicología y Pedagogía Universidad Pedagógica Nacional. Docente orientadora de la Secretaría de Educación Distrital, Bogotá, D.C., Colombia. orcid.org/0000-0003-0144-9865. 
cierre se presentan algunas de las restricciones que experimentan las mujeres negras afrodescendientes, a causa de este racismo epistémico.

Palabras clave: Eurocentrismo; invisibilización en el currículo; marginalización académica; racismo; restricciones en el sistema educativo.

\section{ABSTRACT}

This article presents one of the central findings of the doctoral research: «Educational experiences of Afro-descendant women: An intersectional study», in which the epistemic racism evidenced in the educational experiences of six Afro-descendant women with doctoral studies in Colombia is retaken. This racism is especially revealed in higher education spaces, through three forms: Eurocentric thinking, which gives priority to European knowledge and distorts the epistemes produced from other non-westernized worldviews; invisibility in the curriculum, manifests itself when in the different spaces of formal education the epistemic elaborations different to the Eurocentric parameters are not shown or unknown; academic marginalization is identified through the undervaluation of content, the undervaluation of racialized groups and the underrepresentation of afro-descendant people in academic production spaces. As closing ideas, some of the restrictions that Afro-descendant women experience as a product of this epistemic racism are presented.

Key words: Eurocentrism; invisibility in the curriculum; academic marginalization; racism; restrictions in the educational system.

\section{INTRODUCCIÓN}

Este artículo desarrolla algunas ideas sobre el racismo epistémico evidenciado en las realidades educativas de las mujeres participantes en la investigación doctoral titulada Experiencias educativas de mujeres negras afrodescendientes: Un estudio interseccional. La investigación tuvo como 130 | INTEREDU № 4 VOL. I (JULIO 2021) PÁGS. 129-160. ISSN: 2735-6523 
propósito analizar las experiencias educativas de seis mujeres negras afrodescendientes con estudios doctorales, desde la perspectiva interseccional entre género, raza y clase, tomando como referente los contextos sociocultural y educativo colombiano entre el año 2000 y 2018. La problematización que justificó la investigación está anclada a una realidad que entiende los procesos de exclusión, pobreza y discriminación como consecuencia del racismo estructural; este último ha impactado durante mucho tiempo las condiciones de las comunidades negras afrodescendientes en la región. Las dinámicas y las relaciones jerarquizadas racialmente ubicaron desde la época de la colonia a hombres y mujeres negros en los más bajos peldaños de la estructura social, a pesar de realizar trabajos considerados como la base económica que constituyó la sociedad colombiana y latinoamericana que hoy conocemos (Friedemann y Espinosa, 1995; Rodríguez, 2008; Rodríguez, 2010; Pita, 2012).

Este precario reconocimiento de la comunidad negra afrodescendiente ha trascendido en el tiempo. Se evidencia actualmente en los desactualizados y contradictorios ejercicios censales que tienden a invisibilizar y desconocer el grueso de la población, así como sus necesidades y potencialidades; las desventajas económicas y sociales en las que se encuentran estas poblaciones; o el abandono a los seguimientos normativos pensados para estas comunidades, los cuales tienen lentos y disimulados impactos en el respaldo de sus derechos (Benavides, Torero, Valdivia, 2006; Agudelo, 2010; Gómez, 2016; Granada 2021).

Estas problemáticas afectan de manera contundente a las mujeres negras afrodescendientes, quienes, por su condición de género, tienden a asumir mayores cargas y responsabilidades en los espacios familiares, escolares-académicos y laborales. Situación que, sumada a las dinámicas de estereotipación combinada entre raza y género, ha llevado a que gran 
parte de estas mujeres tengan fuertes limitaciones en el goce de sus derechos, especialmente los referidos al acceso y a la participación en espacios de educación superior, en programas posgraduales (Castellanos, 2011; UNESCO, 2012; Ocoró, 2017). Dicha restricción también abarca las esferas laborales haciendo que se complejicen para ellas los procesos de discusión, debate y aportes a la academia.

Gran parte de los estudios que revelan las situaciones de acceso y participación de las mujeres negras afrodescendientes se centran en comprender las dinámicas de discriminación que permanecen en los distintos espacios y niveles educativos, temáticas que resultan relevantes, pero tienden a la homogeneización de las situaciones educativas de estas mujeres (Hopenhayn y Bello, 2000; Arocha, Guevara, Londoño, Moreno y Rincón, 2007; Soler, 2009; Rodríguez, 2010; Mena, 2011; Castillo y Caicedo, 2012; Ibagón, 2016; Quintero, 2013a). En esta perspectiva se desatienden las experiencias emergentes de mujeres negras afrodescendientes que han transitado y participado en los distintos niveles del sistema educativo, hasta lograr ubicarse en posiciones de importante reconocimiento en sus campos de acción comunitaria, personal y profesional; y de las cuales se pueden identificar factores de oportunidad y restricción para el acceso y la participación en procesos de educación avanzada.

Este aspecto se convirtió en el centro del proceso investigativo, enfocado en analizar y comprender las experiencias educativas de seis mujeres negras afrodescendientes con estudios doctorales. Así, desde una perspectiva interseccional, se identificaron factores de oportunidad y restricción en el acceso, la participación y la culminación de procesos en educación avanzada. Las mujeres de la investigación se autorreconocen como negras afrodescendientes y son doctoras en distintas disciplinas, además se desempeñan en espacios académicos e investigativos en 
diferentes lugares de Colombia y América $^{2}$. Ellas desde sus experiencias aportaron elementos centrales para la comprensión de dinámicas que limitan los procesos formativos de las comunidades negras afrodescendientes, particularmente, las mujeres adscritas a estos grupos.

El estudio se sustentó en los desarrollos teóricos de Silvia Walby (2003, 2007) cuya perspectiva de análisis ontológico interseccional ofrece elementos importantes para la comprensión de las experiencias educativas desde las categorías de identidad o sistemas de relación: raza, género y clase. Luego se retoman elementos cruzados e interseccionales de estas y otras categorías emergentes. Esta apuesta teórica retoma procesos y conceptualizaciones diseñadas a partir de la teoría de la complejidad, que enriquecen las comprensiones interseccionales en tanto que muestran las diferencias entre los sistemas y el entorno; pone en evidencia que un sistema no necesariamente debe saturar un contexto; expone los sistemas como dinámicos y cambiantes, pues no se anclan ni permanecen estáticos; se enfoca en las dinámicas y en las actividades que emergen de la interacción de los sujetos en los contextos determinados; e introduce la importancia de los pequeños eventos como desestabilizadores para los sistemas y pasa de una perspectiva jerarquizada de los sistemas a una coevolución permanente entre ellos (Walby, 2003, 2007).

El corte naturalista, intensivo y en profundidad de la perspectiva metodológica cualitativa permitió un acercamiento a las experiencias de mujeres negras afrodescendientes en sus contextos sociales cotidianos; además de privilegiar el análisis de la información en términos descriptivos, interpretativos y comprensivos de los datos en momentos y lugar determinados (Deslauriers, 2004). El método empleado para el

\footnotetext{
${ }^{2}$ Las seis mujeres participantes aparecen con los siguientes seudónimos: Palma, Perla Negra, Acacia, Ceiba, Ocoba y Michelle.
}

INTEREDU № 4 VOL. I (JULIO 2021) PÁGs. 129-160. ISSN: 2735-6523 
desarrollo de la investigación se inscribió en los estudios biográficos, específicamente, en los relatos de vida (Güereca, 2016) con perspectiva de estudio de caso múltiple particular (Stake, 1999; López, 2013). Las técnicas que permitieron la construcción de la información fueron las entrevistas formales semiestructuradas a profundidad, las charlas informales, revisión documental y diario de campo. Para la organización y análisis de la información se emplearon estrategias manuales y el uso del programa de análisis cualitativo asistido por computadora Nvivo12.

Los hallazgos, las comprensiones y los análisis de la investigación se presentan tomando como guía la propuesta de análisis ontológico de los sistemas de Silvia Walby. A partir de allí se estableció un primer nivel del análisis en el que se comprendió cada categoría o sistema de relación (raza, género, clase) presente en las experiencias de las mujeres. Como segundo momento del análisis, se revisaron el tipo de intersecciones, cruces o relaciones categoriales presentes en las experiencias relatadas, retomando las variaciones, movilidades e impactos que, entre las diferentes categorías, se presentaron.

Al considerar la amplitud de la información construida en la investigación, el presente documento sólo expone uno de los hallazgos centrales en los análisis desarrollados bajo la categoría o constructo social $\mathrm{Raza}^{3}$. En el estudio, esta categoría fue desarrollada de manera profunda abarcando elementos como: los efectos de la categoría racial en el

\footnotetext{
${ }^{3}$ La raza ha sido una categoría útil en procesos de clasificación social que emplea «un borramiento de la historicidad y arbitrariedad de tal clasificación y las relaciones que de esta se derivan» (Restrepo y Rojas, 2010, p.118). Esta idea se traduce en que, al tiempo que se justifican estructuras y relaciones sociales basadas en diferencias fenotípicas, se anulan todo rastro y posibilidad de comprensión histórica de las y los africanos esclavizados. Parte de este borramiento se refiere a la eliminación de «prácticas comunitarias ecológicas, saberes de siembra, de tejidos, del cosmos [...] cambio y control de las prácticas reproductivas y sexuales» (Lugones, 2011, p.108).

134 | INTEREDU № 4 VOL. I (JULIO 2021) PÁGS. 129-160. ISSN: 2735-6523
} 
reconocimiento y autorreconocimiento de las mujeres por sus características fenotípicas (color de piel, textura de cabello, contextura corporal) y sus prácticas étnico culturales; las diferentes formas de discriminación racial que pueden ser efectuadas de manera directa, solapada o naturalizada en los diversos contextos de los cuales hacen parte las mujeres negras afrodescendientes; el endorracismo, el mestizaje y blanqueamiento como mecanismos de la ideología racista que puede operar de manera dinámica y conveniente de acuerdo con las circunstancias y necesidades de algunas de las mujeres participantes; y los estereotipos racializados que vinculados a la prostitución, el servicio doméstico, el deporte, el folclor y la agresividad, ubican en lugares comunes y alejados de la razón, la lógica y la producción de conocimiento a las mujeres y hombres negros afrodescendientes.

Desde esta perspectiva, la Raza como categoría de identidad o marcador diferencial ${ }^{4}$ resultó tener un notable peso e impacto mayoritariamente negativo que ha recaído en las mujeres participantes de la investigación, pues al ser ubicadas racialmente como inferiores en la escala de clasificación social, las pone de entrada en lugares de desventaja, en comparación con otros sujetos que no son racializados como negros afrodescendientes. Este aspecto se evidencia de manera contundente en las prácticas y dinámicas que circulan de manera continua en el sistema educativo y la academia en Colombia y otros países de la región, tal y como se presenta en el análisis relacionado con el racismo epistémico.

De acuerdo con lo explorado en la investigación, el racismo epistémico se expone a través de tres caras o formas de operar: el

${ }^{4}$ El término de categorías de identidad se usó para referir las maneras en las que las participantes se asumen o se adscriben con criterios de género, raza-étnica, clase, edad, lugar de origen; y el término de marcador de diferencia o desigualdad, se empleó para reseñar que los criterios pueden ser asumidos como medio para promover desventajas en el espacio social.

INTEREDU № 4 VOL. I (JULIO 2021) PÁGS. 129-160. ISSN: 2735-6523| 135 
pensamiento eurocéntrico, como forma de determinar las verdades que son legítimas y propias de la academia; la invisibilización en el currículo de los aportes desarrollados desde lo negro afrodescendiente; la marginalización académica presentada a través de la infravaloración de contenidos, la infravaloración de los grupos racializados y la infrarrepresentación de hombres y mujeres negras en los espacios académicos.

\section{LAS TRES CARAS DEL RACISMO EPISTÉMICO EN LOS ESPACIOS DE EDUCACIÓN SUPERIOR}

La estructura que contiene los estereotipos racializados ${ }^{5}$ y que desvincula a las personas negras y a sus comunidades del uso de la razón, del pensamiento y de la producción de conocimiento, se conoce como racismo epistémico. En palabras de Ramón Grosfoguel, se trata de «la inferioridad de todos los conocimientos procedentes de seres humanos clasificados como no-occidentales, no-masculinos o no-heterosexuales» (2013, p.37). Dicha inferioridad se designa por el grupo de sujetos que, autoproclamándose como poseedores de verdades universales y alejadas de las realidades múltiples, clasifican a los sujetos como inferiores no solo

\footnotetext{
${ }^{5}$ Los estereotipos racializados hacen parte de la dimensión «mental» o «cognitiva» del racismo (Van Dijk, 2010 en Quintero, 2013b, p.54), es decir, están anclados a las ideas que se construyen socialmente tomando como referencia las diferencias raciales. Estas ideas tienden a generalizar situaciones, comportamientos, actividades y características asociándolas a las categorías raciales, étnicas o religiosas, sin tener pruebas o evidencias de lo que se plantea (Quintero, 2013b). Los estereotipos racializados vinculados a las mujeres negras afrodescendientes están relacionados con la prostitución, el cuidado y el servicio doméstico, además del desarrollo de actividades folclóricas y deportivas. Si bien la mayoría de estos son presentados en términos positivos sobre la riqueza en las muestras artísticas, costumbres y tradiciones; o por las habilidades atléticas de las personas negras (Meneses, 2014; Vásquez-Padilla, 2019), a través de ellos tiende a repetirse la insinuación que estas son de las pocas áreas en las que mujeres y hombres negros afrodescendientes demuestran sus atributos y capacidades.
}

136 | INTEREDU № 4 VOL. I (JULIO 2021) PÁGS. 129-160. ISSN: 2735-6523 
por su raza, también por su supuesta «incapacidad» de pensar y, por lo tanto, de «existir» (Grosfoguel, 2013). Al tener en cuenta lo aportado por las mujeres de la investigación, las maneras en las que se evidencia el racismo epistémico son: el pensamiento eurocéntrico, la invisibilización en el currículo y la marginalización académica.

\section{PENSAMIENTO EUROCÉNTRICO}

El eurocentrismo es una forma de «creencia en la superioridad de los valores propios y a la vez, como conjunto de características, formas y modos de validez universales» (Busso, 2014, p.1), que da lugar a la hegemonía del pensamiento surgido en Europa, específicamente en cuatro países: Italia, Francia, Inglaterra y Alemania (Grosfoguel, 2013); y que funciona en el territorio europeo, pero trasciende a nivel mundial (Busso, 2014). La priorización del pensamiento europeo se mantiene a lo largo del tiempo a través de las universidades, entendidas como instituciones cuya función es formar, discutir y construir conocimiento. $\mathrm{Al}$ respecto, la experiencia de Palma permite verlo tangiblemente en la educación formal e investigativa:

La gente que estaba en la maestría conmigo solo se pensó en Europa, solo pensaron los griegos, lo que los griegos pensaron y ya, hasta ahí, esa es toda la posibilidad del pensamiento. Entonces no había nadie que pudiera dirigir mi tesis, bueno, casi no encuentro un director para esto, porque no son temas propios de la filosofía. Los temas propios de la filosofía son el poder en Marx, la noción de Ecclesía en Kant, cosas así. Es pensar y repensar sobre lo mismo, es como morderse la cola todo el tiempo. (Palma, entrevista 24 de septiembre de 2018) 
Cuando Palma plantea la inexistencia de quién la pudiera dirigir, se refiere a que, en ese momento, el tema que ella estaba investigando (raza), se encontraba asociado a un área de poca relevancia para el programa de una universidad pública de Cali-Colombia, donde cursaba su maestría. Además, insinúa que, a pesar de la relevancia que han tenido las elaboraciones epistémicas europeas, pareciera que siempre se trabaja sobre lo mismo y no se abren posibilidades para otras formas distintas de pensamiento. Al respecto, Hugo Aníbal Busso lo explica claramente: «el eurocentrismo es un obstáculo epistemológico para el análisis, la explicación y la interpretación de las transformaciones sociales, culturales y políticas» (2014, p.2). Este obstáculo epistemológico que caracteriza el eurocentrismo en las universidades latinoamericanas, o para ser más específicos, en las colombianas, nos revela que, pareciera que las personas racializadas «no se encuentran» al mismo nivel de producción de conocimiento propuesto por los esquemas de la academia europea. Esto no se debe a las capacidades que tengan o no para hacerlo, sino que, de entrada, ya son considerados inferiores por su raza y prácticas culturales. Al respecto, Perla Negra relata que su tutor de tesis doctoral, un hombre blanco alemán, le hace a ella y a una de sus compañeras un llamado de atención sobre su forma de escritura:

Un día yo me fui con Elvia ${ }^{6}$ a hablar con el profe y le dije: «profe yo vengo aquí con Elvia y - ella estaba preocupada - ella no consigue avanzar y usted no le lee», y el profesor responde: «mire, les voy a decir una cosa y es para las dos, Elvia y usted escriben como negros y la academia es blanca, ese es el problema, ustedes no escriben como blancos». (Perla Negra, entrevista 4 de octubre de 2018)

\footnotetext{
${ }^{6}$ Se cambió el nombre original para respetar el anonimato acordado con las participantes de la investigación.

138 | INTEREDU № 4 VOL. I (JULIO 2021) PÁGS. 129-160. ISSN: 2735-6523
} 
La guía dada por el docente director de tesis doctoral de dos mujeres negras en una universidad de Brasil se orienta hacia el blanqueamiento euroepistémico, es decir, busca eliminar los vestigios de la diversidad epistémica (Grosfoguel, 2013) percibida en sus formas de escritura. Podría pensarse desde este relato que, dentro del esquema eurocéntrico con el que fueron fundadas nuestras universidades, se sustenta y se defiende que un sujeto europeo forme a otros sujetos - racializados - no europeos, bajo los parámetros y esquemas con los que fue educado en el epicentro del conocimiento «universal» europeo. Es más, este patrón se repite con sujetos - hombres y mujeres - no europeos, pero que formados bajo líneas de pensamiento eurocéntricos se consideran autoridad para determinar lo que es inferior o superior, epistémicamente hablando.

Esto quiere decir que, varias de las universidades, a través de sus docentes, repiten a mediana y pequeña escala el racismo epistémico con aquellos estudiantes que poseen otras cosmovisiones y formas de pensamiento. Al respecto, Palma nos lleva a reflexionar cuando presenta a la universidad como una entidad que, en sus dinámicas cotidianas, resulta de difícil comprensión y asimilación, especialmente para los sujetos que culturalmente se han formado lejanos a principios académicos de occidente.

La universidad es un ambiente que me acerca a mí con lo académico, con las letras, con los libros, entonces a mí no se me hace difícil, pero se piensa debe ser difícil para mí, ¿no? Y de hecho lo es para la gente que no está socializada en este mundo tan occidentalizado. Lo es y lo hemos visto en algunos docentes en su relación con comunidades indígenas y comunidades afro, pero no porque los estudiantes de estas comunidades sean inferiores intelectualmente, pero los profesores siendo científicos sociales lo asumen así. Lo digo porque 
lo he oído en colegas. en reuniones en la universidad, quejándose del desempeño académico de los estudiantes negros y de los estudiantes indígenas. no hay una explicación, simplemente asumen que «son malos estudiantes». Es como un dolor de cabeza porque su desempeño es muy bajo, entonces no asumen que para los indígenas el español, por lo general, es como la segunda lengua, que ambas culturas [indígena y afro] son culturas de tradición oral, en donde la oralidad es lo más fuerte. (Palma, entrevista 24 de septiembre de 2018).

De acuerdo con lo relatado por Perla Negra y Palma, ser negro o negra las ubica en un lugar de desventaja dentro de la academia o en los procesos de producción de conocimiento, pues al parecer «carecen» de los elementos requeridos para ser académico «blanco» $y$, por tanto, crear como «blanco». Ahora, otro de los aspectos que se revelan en el eurocentrismo como obstáculo en la academia y que está muy relacionado con el elemento anterior, es la insistencia que manifiestan los sujetos racializados como «inferiores» para ser parte del grupo euroepistémico, a pesar de no encajar en los patrones construidos por los europeos. Uno de estos modelos se refiere a la búsqueda a toda costa de la idea de «progreso»y «desarrollo» (Busso, 2014) en la academia. Por ejemplo, Palma nos cuenta que, desde su percepción:

Los pregrados y las maestrías de las universidades colombianas parten de Europa, o sea, se asume que aquí colonizados no estamos, que aquí ya estamos es en el camino a la modernidad y que ser modernos es el camino, o sea, es a donde debemos llegar y que todos nuestros problemas son porque no somos modernos como son los europeos. Entonces, frente a eso ¿qué?, o sea, la discusión es simplemente cómo nos hacemos modernos, es que esa es en últimas la discusión, necesitamos ser modernos, el modelo es Europa, por eso es eurocéntrica esta academia. (Palma, entrevista 28 de septiembre de 2018). 
Siguiendo esta línea, en el horizonte del «desarrollo»y del «progreso», la academia debe estructurar sus planes y estrategias pedagógicas para responder a los intereses eurocéntricos, es decir, sus principios orientadores serán determinados por las necesidades y los cambios sociales, económicos y culturales a nivel global, más no local o regional.

Ese racismo estructural pasa en la universidad por los pénsum académicos, por los currículos; no sólo por cómo te miran, sino también por aquello que se pone en el pénsum, a qué autores se lee [...] Porque no se lee a Fanon, por ejemplo, nunca se lee a Césaire. Es decir, a los autores negros no se los leen, porque como que no tienen que aportar. En filosofía recuerdo a un profesor, cuando alguien mencionó a Fanon, el profesor decía que era como muy sangriento, muy violento, como que daba susto las propuestas del tipo. Entonces, no se le reconoce un discurso filosófico a Fanon sino que se lo juzga como un hombre violento, de una propuesta violenta. Pero a Marx no se lo trata de la misma manera, no se lo trata igual cuando su propuesta también es fuerte, pues habla de la lucha de clases y como eso no se resuelve a las buenas, porque a las buenas no se puede resolver y aun así se le sigue leyendo. Entonces, hay un tratamiento muy diferenciado en los currículos, pero no sólo un tratamiento diferenciado, es más una negación para reconocer que hay un pensamiento que vale la pena ser leído, o sea, no hay un reconocimiento del hombre y la mujer negros como interlocutores válidos en una discusión de orden epistemológico, como si no tuviesen nada que aportar. Eso es muy visible. (Palma, entrevista 28 de septiembre de 2018). 


\section{INVISIBILIZACIÓN EN EL CURRÍCULO}

La priorización de las temáticas, los autores que se leen y se estudian, las maneras en que se abordan las reflexiones en busca de la producción de conocimiento ponen en evidencia no solo el tipo de pensamiento que se desea mostrar y reforzar, también aquel que se quiere omitir o eliminar. En otras palabras, se realiza una destrucción de otras epistemes o «epistemicidio» (De Sousa Santos, 2010) solapado, ejercido a través de la invisibilización de las prácticas, formas de conocimiento diversas a las eurocéntricas. Así pues, cuando la academia no muestra o desconoce lo que las «otras» epistemologías han elaborado, alejados de los parámetros academicistas eurocéntricos, se puede decir que se efectúa un racismo epistémico por invisibilización.

$\mathrm{Al}$ respecto, Palma afirma que, en diferentes comunidades, ubicadas en el Pacífico colombiano, permanecen vigentes múltiples formas de socialización, construcción de comunidad, excluidas de los textos o de los programas académicos de las universidades del país:

Hay un parentesco espiritual ahí, que ya no lo disuelve nada, absolutamente nada, y hay una obligatoriedad, un respeto, una solidaridad entre comadres y compadres que es más fuerte que lo consanguíneo. Entonces ¿cómo puede pasar eso?, eso ha pasado por alto todos estos años, ha sido invisibilizado, no se ha visto y tiene una fuerza tan impresionante, es súper importante. Y todo eso está desapareciendo también por el impacto de la modernidad [...] por el desarrollo, está desapareciendo. Y no es desaparecer el folclor, es desaparecer formas alternativas de vida, que son una respuesta a toda esta crisis civilizatoria en que nos tiene Occidente. (Palma, entrevista 24 de septiembre de 2018) 
Como estas formas diferentes y diversas de conocimiento no cumplen con las características de un pensamiento eurocéntrico, no son consideradas por el mundo moderno como episteme o conocimiento experto, que hace parte del mundo de las ideas; más bien son entendidas como doxa o conocimientos basados en creencias, opiniones e imaginación (Piñeiro, 2017).

Al estar incorporada la idea de superioridad epistémica "blanca" (Arocha et al., 2007) se niegan las elaboraciones otras que no cumplen con los patrones establecidos, dejando en evidencia el poco impacto o desarrollo de los procesos etnoeducativos en el país. Así pues, se pierde la posibilidad de reconocer los aportes de diferente índole desarrollados por sujetos y colectividades negras afrodescendientes (Romero, 2010; Meneses, 2013; García, 2011, 2019). La invisibilización epistémica se manifiesta de distintas maneras: una de ellas, es en los textos académicos cuando no se muestran elementos de tipo gráfico o narrativo que vinculen lo negro afrodescendiente con la producción de conocimiento (Arocha et al., 2007; Soler, 2009), o cuando no se exponen los aportes y el papel de las poblaciones racializadas en la construcción de nación (García, 2015).

Un amigo historiador me recomendó un libro [...], me dijo: «que era muy bueno», fui y lo compré, se titula «Historia mínima de Colombia». Entonces, allí uno encuentra desde los precolombinos, los primeros habitantes de los 12.000 hasta los 3.000 A.C., entonces súper chévere. Es un compendio, como una pequeña enciclopedia, entonces yo voy a buscar qué dice sobre la población negra y encuentro tres líneas, yo dije: «pero ¿cómo así, esto qué es?». Miro en el capítulo sexto la liberación de los esclavos y la igualdad de los indios, «ah, la liberación», no, pues, habla tres líneas de la liberación de los esclavos y luego habla de los indios y se acabó el tema. Entonces ¿qué es lo bueno que tiene esta historia? O sea, es la misma historia institucional, la misma historia blanca, la misma historia de INTEREDU № 4 VOL. I (JULIO 2021) PÁGs. 129-160. ISSN: 2735-6523| 143 


\section{Lubi Jehins Granada Angulo}

las élites en donde no aparece la población indígena, la población negra, ni sus luchas aparecen. Entonces, yo estaba pensando, ¿esto qué tiene de bueno, en últimas, cuando es la misma historia?, realmente no me dice nada. No, es chocante, me encuentro ese tipo de cosas supremamente chocantes porque tanto que se ha dicho ya, tanto que se ha discutido, que se ha planteado la invisibilización en la historia de la población negra y los historiadores simplemente siguen haciendo oídos sordos a esos asuntos, siguen escribiendo como si de eso no se hubiera dicho nada, entonces, pues resulta supremamente chocante (Palma, entrevista 28 de septiembre de 2018).

Otra forma de invisibilización epistémica se da perversamente con la castración de la libertad de cátedra impartida por una mujer docente negra y doctora, en una universidad privada en Bogotá, quien desea educar tomando también como referentes sus producciones académicas:

[...] A veces yo me siento prostituida por dos pesos, porque muchas de las cosas que tú piensas y escribes, tú no las puedes reproducir en el aula de clases, porque lo que tú estás diciendo no va. Porque si hablo de los negros, no, entonces se piensa que «solo en mi clase se habla de negros o de mujeres», cosa que no es cierta. Pero tú sabes que hay una campaña de descrédito [por estos temas] y así lo generalizan, por eso toca reproducir el discurso tradicional (Perla Negra, entrevista 2 de octubre de 2018).

Los relatos de Palma y Perla Negra evidencian la invisibilización de las epistemes diversas en la academia colombiana, ejercidos a través de los textos académicos y la «libertad de cátedra». Además, muestran la inconformidad y la molestia que genera sentir que, a pesar de los esfuerzos para que sean reconocidos formalmente los aportes y 
construcciones de pensamiento de las comunidades negras, solo pueden resignarse a ser considerados como elaboraciones marginales.

\section{MARGINALIZACIÓN ACADÉMICA}

La marginalización es otra de las maneras en la que el racismo epistémico recalca la inferioridad en la construcción de pensamiento, donde "al no poder incluirse como "corpus", es relegado a un segundo plano del conocimiento» (Piñeiro, 2017, p.70). Hecho que se entiende en la acción pedagógica fundamentada desde la universidad eurocéntrica, la cual es principalmente «desautorizadora», pues «nos declara ineptos, nos impide producir categorías de impacto global» (Segato, 2013 en Piñeiro, 2017, p.70). Retomando las experiencias de las participantes en la investigación, se pueden caracterizar tres formas de marginalización académica: infravaloración de contenidos, infravaloración de los grupos racializados y la infrarrepresentación de mujeres y hombres negros en los espacios de producción académica.

La infravaloración de contenidos consiste en poner en segundo o tercer plano todas las elaboraciones referidas a los temas raciales y étnicos, puede hacerse visible a través del no reconocimiento de la importancia que tienen estos temas en la academia. Acacia ilustra cómo este tipo de marginalización se hizo presente en su experiencia académica e investigativa, cuando, luego de regresar de terminar su doctorado en Estados Unidos, se presentó a una convocatoria de investigación en una universidad pública colombiana y al exponer su temática investigativa, la cual relacionaba el racismo y las matemáticas, recibió una fuerte respuesta:

Yo, por ejemplo, me presenté a una convocatoria de una universidad pública en Santander-Colombia y fue una experiencia súper dolorosa. Porque casi que me dicen: usted está loca, a usted ¿quién le dijo que eso realmente es investigación en el campo de la INTEREDU № 4 VOL. I (JULIO 2021) PÁGS. 129-160. ISSN: 2735-6523| 145 
educación matemática? Yo me acuerdo de que estuve en la cama casi como tres días deprimida antes de poder como asimilar la experiencia de presentarme en esa universidad, fue horrible. (Acacia, entrevista 14 de noviembre de 2018).

El relato de Acacia orienta la reflexión hacia la respuesta negativa que tiene al trabajar los temas raciales en la academia, situación que se refuerza aún más cuando dichos contenidos están relacionados con las ciencias «puras» o «exactas», los cuales suponen una distancia con el mundo de las realidades y problemáticas sociales. La no apertura de estas necesidades epistémicas en la academia, además de limitar la construcción del pensamiento desde otras posibilidades, genera desmotivación en los sujetos interesados en aportar desde diversas perspectivas. Aunque el desinterés también pareciera estar presente en las personas no racializadas como negras, pues lo ven como algo que no genera mayor problema y que no toca sus fibras de humanidad.

No hay un interés real por los temas [raciales y étnicos], a nadie le interesa realmente las temáticas, la gente toca las temáticas, pero salen fácil de ellas, porque la gente dice: pero si al final eso es un tema que a la gente no le importa. ¿Y por qué no le importa? Uno de los razonamientos que yo hice en mi tesis doctoral es que a la gente no le importa porque es una condición que, como usted no nació así «negro», entonces usted nunca va a ser así, entonces nunca le va a tocar el problema (Ceiba, entrevista 10 de octubre de 2018).

Como segunda forma de marginalización epistémica, está la infravaloración de los grupos racializados a través de los contenidos, ya sea porque se folclorizan y descontextualizan en su implementación (Meneses, 2013; Moreno, 2014) o porque se ignoran completamente. Es 
decir, este tipo de marginalización se evidencia directamente en cómo las personas negras no se encuentran representadas en los programas académicos de las instituciones educativas a las cuales asisten, haciendo que se disipen en los espacios de educación formal:

Lo triste también es que esa formación [académica], es una formación que hace que la gente termine perdiéndose, porque la gente indígena y la gente negra no va a encontrar allí cómo reforzar sus raíces identitarias. Por ejemplo, mira el libro del que te hablé, de la Historia mínima de Colombia, pues para hablar de Bolívar otra vez, de las luchas emancipadoras, pues del siglo XIX, de los conflictos entre liberales y conservadores, para volver a hablar de eso. ¿Dónde están las luchas indígenas? ¿Dónde están las luchas negras y toda la historia cimarrona? O sea, eso es muy marginal, si aparece, aparece de manera muy marginal (Palma, entrevista 28 de septiembre de 2018).

La tercera forma de marginalización académica es la infrarrepresentación de las personas negras en espacios de producción de conocimiento. Si bien la infrarrepresentación puede ser entendida como el bajo porcentaje de cargos ocupados por personas negras en los espacios de formación, investigación y dirección (Viveros, s.f.; Silva, 2010), también nos lleva a pensarla como la no valoración, poca visibilidad o no reconocimiento de los aportes, logros y esfuerzos realizados por los sujetos racializados como negros en los espacios académicos (García, 2015). A través de las experiencias de las mujeres participantes en la investigación, se puede evidenciar cómo la infrarrepresentación toma múltiples formas en escenarios diferentes. Por ejemplo, en los espacios de debate, reflexión y construcción de conocimiento, se llega a no reconocer el título de educación avanzada. 
Por ejemplo, cuando yo tuve mi primera sustentación de invitada como doctora, estaba haciendo el postdoctorado $-\mathrm{y}$ en varios escenarios ha pasado, porque se ve en muchos escenarios-, entonces presentaron a los doctores y a mí me presentan como la doña. En Brasil se dice mucho «doña», «estoy presentando a doña Perla Negra», ¿doña Perla Negra? Los otros son doctores y yo soy doña. Ahí sí me sentí, y le dije: «ay profe perdón, yo estudio mucho pa' no ser doña, doña Perla Negra no, yo también soy doctora, doctora Perla Negra» [...] Claro, en frente de todos en ese momento, fue una cosa impresionante. El profesor dijo: «ay, disculpe es una forma de decir» y yo, no, doña no, y «nada contra las doñas, con las señoras que están en ese ambiente porque las respeto, pero yo no quise ser ama de casa pa' que tú me trates así» (Perla Negra, entrevista 4 de octubre de 2018).

La situación expuesta por Perla Negra manifiesta que, a pesar de encontrarse en un espacio con pares académicos del mismo nivel de formación, fue presentada públicamente como una mujer sin créditos formativos, marginalizando los méritos que la llevaron a ubicarse en ese lugar en el campo educativo. De manera similar, se pueden encontrar experiencias en los espacios laborales donde el reconocimiento a su formación es invisibilizado:

En la institución donde yo trabajo, cuando hay las ceremonias de graduación, hay la dificultad que todos en el equipo directivo no son doctores, entonces cuando sucede esto, a todos nos presentan como magíster. Para no reconocer ante la comunidad educativa la realidad de que una directiva negra sea la única doctora de la institución (Ceiba, entrevista 9 de octubre de 2018). 
Este asunto permite pensar que, en dichos casos, a la academia y sus representantes les cuesta reconocer que estas mujeres, al igual que otros sujetos, tuvieron las capacidades y posibilidades para ser doctoras y ubicarse en los lugares en los que se posicionan. Este aspecto referido al lugar que se ocupa, se enfocaría más bien al lugar que no debería estarse ocupando, pues al ser un sujeto racializado «como inferior», resulta poco normal, o común, encontrarse en un lugar que no es el lugar (Grosfoguel, 2013) en el que siempre fueron ubicados históricamente, y es el lugar de la pobreza, la pereza, la hipersexualización, el folclor, el deporte y la ignorancia (Lozano, 2010; Morales, 2012; Hellebrandová, 2014; Meneses, 2014; Moreno, 2014; Vásquez-Padilla, 2019).

En la academia, [...] lo reafirmo, sobre todo, cuando se trata de estudios en los cuales ya se va subiendo el nivel en formación avanzada, sobre todo, entonces ahí es explícito las infravaloraciones de tu presencia en espacios de formación y de enseñanza, es una infravaloración, es un desprecio total y es el hecho de decir pues el fenómeno "mosco en leche", “¿qué hace esta, aquí?", así de sencillo. (Ceiba, entrevista 10 de octubre de 2018).

Por ejemplo, en mi caso si vos le preguntas a un grupo de personas cualquiera aquí en Estados Unidos, incluyendo negros, les preguntas: ¿usted cuál cree que es el máximo título alcanzado por Michelle? Por ahí el $70 \%$ te va a decir probablemente de nivel técnico, la gente todavía no cree que yo pueda ser doctora (silencio prolongado). (Michelle, entrevista 17 de noviembre de 2018).

Ante esta infrarrepresentación, varias de las mujeres expresan que ellas o personas conocidas deben optar por estrategias para que socialmente en el interior de la academia o en sus espacios laborales les sean reconocidos sus esfuerzos y logros educativos. Para ilustrar este argumento, se cita uno de los relatos de Michelle, quien comenta cómo 


\section{Lubi Jehins Granada Angulo}

una amiga de ella, que también es negra, debe ubicar su título de doctora en diferentes documentos, sólo por el hecho de hacer reconocer su formación académica y ser tratada de manera adecuada:

Mi amiga africana me dice que ella cuando viaja se pone su título, o sea ella en el pasaje aparece como Doctor Elena Bantu ${ }^{7}$. Porque ella me estaba diciendo que nadie cree que ella es doctora, entonces ella se lo pone en su tiquete. Ella se lo pone para que las personas no asuman que ella es una persona que no es educada, o que pueden pasar fácilmente por encima de ella. (Michelle, entrevista 17 de noviembre de 2018).

Los actos de infrarrepresentación, evidenciados en los relatos de las mujeres negras afrodescendientes entrevistadas, ponen de manifiesto que la marginalización del racismo epistémico tiene muchas formas de obrar e impactar en la vida de los sujetos racializados. Estas dinámicas pueden llegar a explicarse como hechos aislados que responden a situaciones que, para los no afectados, son pasajeras, inofensivas o sencillamente producto de la ingenuidad o distracción del que las efectúa. Empero, son, en realidad, efectos constitutivos de un racismo epistémico presentes en nuestras sociedades y que se entrevén mediante el eurocentrismo, la invisibilización y las distintas formas de marginalización.

Para finalizar este apartado, se presenta uno de los episodios que revela los alcances del racismo epistémico experimentado por una de las mujeres participantes. En el relato se expone cómo en un evento de clausura y reconocimiento a los esfuerzos académicos, se invisibilizan los logros de dos doctores negros afrodescendientes en una importante universidad del país.

\footnotetext{
7 Se cambió el nombre original para respetar el anonimato acordado. 150 | INTEREDU № 4 VOL. I (JULIO 2021) PÁGS. 129-160. ISSN: 2735-6523
} 
El incidente fuerte que nos sucedió a un compañero del doctorado y a mí fue en el acto de graduación en una universidad en PastoColombia, éramos cuatro graduandos: dos profesores de esta universidad, que estudiaron con beca y con todo (pausa) y nosotros dos, mi compañero [también negro] y yo, que no estábamos haciendo todo financiado sino con recursos propios. Llega el acto de graduación, los dos teníamos tesis doctorales laureadas y los otros dos compañeros que son docentes de esta universidad no tenían nada laureado. El rector había firmado la resolución de laureado de cada una de las tesis y la secretaria general también lo sabía, porque lo había firmado y tuvieron la grosería e ilegalidad de incumplir una resolución. El acuerdo decía que en la ceremonia de grado se debía hacer reconocimiento público por las tesis laureadas de mi compañero y la mía. El día del evento nos la entregaron y no dijeron nada, entregaron las tesis de mérito a los de pregrado, a los de maestría, a los de especializaciones y las nuestras las entregaron ahí sólo con el título, ese fue el acto. Luego obviamente hubo reacciones, pero ese es el acto de discriminación étnico-racial más fuerte en la academia que yo he vivido y que ha vivido mi compañero, porque él no lo pasa todavía y yo tampoco lo paso, pero estamos lidiando con ello (Ceiba, entrevista 10 de octubre de 2018).

\section{IDEAS DE CIERRE}

A lo largo del artículo se evidenció que, diferentes hechos referidos al pensamiento eurocéntrico, la invisibilización en el currículo y las diversas formas de expresión de la marginalización académica son las tres caras del racismo epistémico, el cual hace parte de las dinámicas de varias universidades, espacios de formación y escenarios laborales en Colombia y otros países de la región. Los impactos que tiene la estructura racista en las epístemes diversas afectan de manera colectiva a las INTEREDU № 4 VOL. I (JULIO 2021) PÁGS. 129-160. ISSN: 2735-6523| 151 
comunidades negras, en tanto que no son considerados sujetos válidos en la construcción de conocimiento y su difusión en los textos académicos. Además, de manera individual los efectos en las vidas de las mujeres negras afrodescendientes se revelan en la infravaloración y en la infrarrepresentación, que se establecen sobre sus logros y esfuerzos.

Respecto a la comprensión de la racialización en las experiencias educativas, es notable la connotación negativa que esta tiene en las mujeres participantes de la investigación, pues no se sienten consideradas como sujetos centrales o de referencia en los distintos procesos académicos de los cuales hacen parte. De acuerdo con lo evidenciado, las razones de esta situación se ubican en las configuraciones racistas representadas en estereotipos racializados que socialmente se establecen y se reproducen sobre las personas negras afrodescendientes a quienes no se vinculan con la construcción de episteme o conocimiento.

Es importante resaltar que las restricciones o limitaciones que las participantes puedan experimentar y otras mujeres en condiciones raciales similares, no sólo son parte del sistema educativo: provienen de otros sistemas o dominios institucionales - familiar, político, social, comunitario, económico, entre otros - (Walby, 2007) que, en relación permanente, procrean, configuran y transfieren entre ellos las restricciones hacia las mujeres negras afrodescendientes.

Retomando los hallazgos de la investigación, las restricciones pueden organizarse en tres grupos: restricciones por subestimación, restricciones por negligencia u omisión y restricciones por acto directo.

Las restricciones por subestimación están relacionadas con procesos en los que se demeritan los asuntos, que cruzan las necesidades de estas mujeres asociados a la raza, la etnia y el género. Este tipo de limitaciones se sustenta en que dichos asuntos no son reconocidos como problemáticas que le atañen al general de la población, por lo tanto, se perciben como 152 | INTEREDU № 4 VOL. I (JuLIO 2021) PÁGs. 129-160. ISSN: 2735-6523 
temas marginales y sin importancia. Ejemplo de estas restricciones son: nulo o ínfimo reconocimiento de las discriminaciones de género y racial o la naturalización de este tipo de prácticas en los diferentes contextos, desinterés institucional hacia este tipo de temáticas y problemáticas, poco interés en la atención y seguimiento a las situaciones y denuncias sobre prácticas de discriminación racial y de género, entre otras.

Las restricciones por negligencia u omisión dejan a su suerte a las mujeres negras afrodescendientes. En estas situaciones, aunque conociendo las problemáticas y las normativas que respaldan a estas mujeres, las instituciones frecuentemente hacen caso omiso a este tipo de necesidades. La institucionalidad por sí sola no provee ni garantiza las herramientas para que estas mujeres enfrenten los distintos tipos de discriminación y situaciones de desventaja social. Cuestiones como la desatención en temas de discusión académica y educativa que vinculen la racialización, la feminización y la territorialización de la pobreza; la no implementación de la Cátedra de Afrocolombianidad de obligatorio cumplimiento en las instituciones educativas, tal y como aparece en el Decreto 1122 de 1998 (MEN, 1998:12); la reducida apertura a temas raciales y de género en espacios académicos como seminarios, clases y talleres; ${ }^{8}$ el descuido en el reconocimiento de la representación de mujeres negras afrodescendientes en los espacios educativos como estudiantes, docentes, directivas e investigadoras, se convierten en ejemplo de limitaciones por negligencia u omisión por parte del sistema educativo.

\footnotetext{
${ }^{8}$ Este tipo de restricciones puede ser más evidente en unas disciplinas del conocimiento que en otras. Por ejemplo, en áreas propias de las humanidades y las ciencias sociales son cada vez más frecuentes este tipo de discusiones; sin embargo, en el caso de las ciencias naturales, ingenierías, matemáticas y afines, estos temas tienden a ser poco desarrollados.
} 
Las restricciones por acto directo son aquellas configuradas a través de palabras, comportamientos e intenciones negativas, con la pretensión de ubicar a las mujeres negras afrodescendientes en posiciones de desventaja en el espacio social y en el campo educativo, en particular. Este tipo de limitaciones, empleadas como medio para establecer y mantener dominación sobre estas mujeres, también son estratégicamente funcionales para demarcar sus lugares y formas de participación. Ejemplo de estas son: la configuración y la reproducción de estereotipos racializados y feminizados; la violencia de género en todas sus manifestaciones; la discriminación racial directa y explícita; la invisibilización-marginalización de epistemes feminizadas y racializadas en los espacios educativos y académicos.

Los tres tipos de restricciones están vinculados entre sí, se alimentan y se fortalecen recíprocamente, es decir, coevolucionan. Su característica común predominante está relacionada con la imposibilidad que tienen para transformarse en oportunidades, lo que indica que, por donde se les mire, generan desigualdad en términos de desventajas para las mujeres que las experimenta. Ahora bien, el único vestigio positivo de este tipo de restricciones es cuando se convierten en temas que convocan y movilizan a las mujeres negras afrodescendientes para su comprensión, discusión y resistencia.

Como ejemplo de este aspecto, se presentan las experiencias de estas seis mujeres, quienes lograron superar las bajas expectativas que sobre ellas se efectuaron, proyectando y ampliando un interesante horizonte para otras que, como ellas, contemplen, en el campo de la educación formal, un camino prometedor y restaurador; pero queda en evidencia que al sistema educativo, especialmente a nivel superior, le cuesta evidenciar, comprender y atender los cambios sociales que 
movilizan a los sujetos, esencialmente a estas mujeres que, en resumidas cuentas, también le dan vida a este sistema.

\section{REFERENCIAS BIBLIOGRÁFICAS}

Agudelo, C. (2010). Movilizaciones afrodescendientes en América Latina.

Una visión panorámica de algunas experiencias contra la exclusión y por el derecho a la identidad. Colombia Internacional, 71, 109-126.

Arocha, J., Guevara, N., Londoño, S., Moreno, L. y Rincón, L. (2007). Elegguá y respeto por los afrocolombianos: una experiencia con docentes de Bogotá en torno a la Cátedra de Estudios Afrocolombianos. Revista de Estudios Sociales, 27, 94-105.

Benavides, M., Torero, M. y Valdivia, N. (2006). Más allá de los promedios. Afrodescendientes en América Latina. Washington: The International Bank for Reconstruction and Development/ The World Bank.

Busso, H. A. (2014). El eurocentrismo como obstáculo epistemológico en las ciencias sociales. Posibilidades y desafíos actuales de la filosofía latinoamericana. Saskab. Revista de Discusiones Filosóficas Desde Acá 7. http://www.ideaz-institute.com/sp/CUADERNO7/C74.pdf

Castellanos, G. (2011). La categoría de género y la educación superior: Una mirada a América Latina desde Colombia. La manzana de la discordia, VI(2), 25-40.

Castillo, E. y Caicedo, J. A. (Marzo de 2012). Yo no me llamo negrito. Ponencia presentada en la Conmemoración Día Mundial contra el Racismo «Por una educación libre de discriminación: del reconocimiento formal a la garantía real». Bogotá, Colombia.

Congreso de la República de Colombia. (1998, 18 de junio). Decreto 1122 de 1998. Ministerio de Educación Nacional. Colombia. Diario Oficial No. $43325 . \quad$ http://www.suinjuriscol.gov.co/viewDocument.asp?ruta=Decretos/1861091 
De Friedemann, N. y Espinosa, M. (1995). Las mujeres negras en la historia de Colombia. En M Velásquez (Ed.). Las mujeres en la historia de Colombia. Tomo II mujeres y sociedad (pp. 32-76). Bogotá: Editorial Norma.

Deslauriers, J. P. (2005). Investigación cualitativa. Pereira: Editorial Papiro.

De Sousa Santos, B. (2010). Epistemologías del sur. México: Siglo XXI.

García, J. (2011). La etnoeducación afro "casa adentro". Un modelo político-pedagógico en el Pacífico colombiano. Pedagogía y Saberes (34), 117-121.

-------(2015). Pensamiento educativo afrocolombiano. De los intelectuales a las experiencias del movimiento social y pedagógico. Revista Colombiana de Educación, 159-182.

--------(2019). Políticas públicas de educación afrocolombiana: el arte de escamotear el derecho de los pueblos. Roteiro, 44 (1), 1-20.

Gómez, P. (2016). Indios, negros y otros indeseables. Capitalismo, racismo y exclusión en América Latina y el Caribe. Bogotá: Icono.

Granada, L. (2021). Experiencias educativas de mujeres negras afrodescendientes. Un estudio interseccional. [Tesis de Doctorado, Universidad Pedagógica Nacional]. Repositorio InstitucionalUniversidad Pedagógica Nacional. http://hdl.handle.net/20.500.12209/12568

Grosfoguel, R. (2013). Racismo/sexismo epistémico, universidades occidentalizadas y los cuatro genocidios/ epistemicidios del largo siglo XVI. Tabula Rasa 19, 31-58.

Güereca, R. (2016). Guía para la investigación cualitativa: etnografía, estudio de caso e historia de vida. México D.F: Lerna.

Hellebrandová, K. (2014). Escapando a los estereotipos (sexuales) racializados: el caso de las personas afrodescendientes de clase media en Bogotá. Revista de Estudios Sociales (49), 87-100.

156 | INTEREDU № 4 VOL. I (JULIO 2021) PÁGs. 129-160. ISSN: 2735-6523 
Hopenhayn, M. y Bello, Á. (2000). Tendencias generales, prioridades y obstáculos en la lucha contra el racismo, la discriminación racial, la xenofobia y las formas conexas de intolerancia. América latina y el caribe. Seminario regional de expertos para américa latina y el caribe sobre medidas económicas, sociales y jurídicas para luchar contra el racismo, con referencia especial a los grupos vulnerables. Santiago de Chile, 25 al 27 de octubre de 2000.

Ibagón, N. (2016). Entre ausencias y presencias ausentes. Los textos escolares y el lugar de lo negro en la enseñanza de la historia de Colombia, 19912013. Bogotá: Editorial Pontificia Universidad Javeriana.

Lozano, B. (2010). El feminismo no puede ser uno porque las mujeres somos diversas. Aportes a un feminismo negro decolonial desde la experiencia de las mujeres negras del Pacífico colombiano. La manzana de la discordia, $V(2), 7-24$.

López, W. (2013). El estudio de casos: una vertiente para la investigación educativa. Educere, 17(56), 139-144.

Lugones, M. (2011). Hacia un feminismo descolonial. La Manzana de la Discordia, 6(2),105-119.

Mena, M. I. (2011). El lugar del racismo y la discriminación racial en las memorias de la afroeducación. Revista Pedagogía y Saberes, (34), 105-114.

Meneses, Y. (2013). Representaciones sociales sobre etnoeducación y cátedra de estudios afrocolombianos en la formación del profesorado. Enunciación, 18(1), 45-63.

-(2014). Representaciones sociales sobre afrodescendientes: la aventura cultural, la violencia sexual-género y luchas multidimensionales. Memoria y sociedad, 18(37), 76-92.

Morales, E. (2012). UCLA Electronic Theses and Dissertations. http://escholarship.org/uc/item/6jr3v45n 
Moreno, G. (2014). Representaciones sociales de los estudiantes afrocolombianos en torno a la construcción de su etnicidad. Revista Escuela Administración de Negocios (76), 193-201.

Ocoró, A. (2017). Afrocolombianos y Educación Superior. Un análisis de la participación de las mujeres y los hombres en el sistema de cuotas o condiciones de excepción para las comunidades negras o afrocolombianas en Cali-Colombia (2004-2016). En Daniel Mato (ed.). Educación Superior y Pueblos Indígenas y Afrodescendientes en América Latina: políticas y prácticas de inclusión, democratización e interculturalización. Universidad Nacional de Tres de Febrero.

Piñeiro, E. (2017). Ciencias subalternas y conocimientos otros: una reflexión epistémica desde la Modernidad. RELASO, 7(2), 64-76.

Pita, R. (2012). El trato a los esclavos durante la independencia de Colombia: rupturas y continuidades en una etapa de transición política. Boletín de Historia y Antigüedades, 99 (854), 81-121.

Quintero, O. (2013a). El racismo cotidiano en la universidad colombiana desde la experiencia vivida por los estudiantes negros en Bogotá. Universitas humanística, 77, 71-94.

(2013b) Racismo y discriminación en la universidad: lecturas cruzadas de las sociedades francesa y colombiana a partir de la experiencia vivida por estudiantes negros en París y Bogotá. Tesis Doctoral en Sociología. Université Rennes 2. Español. NNT: 2013REN20009

Restrepo, E. y Rojas, A. (2010). Inflexión decolonial: fuentes, conceptos y cuestionamientos. Popayán: Universidad del Cauca.

Rodríguez, G. (2008). Continúa la exclusión y la marginación de las comunidades negras colombianas. Revista Diálogos de Saberes, 29, 215-238.

158 | INTEREDU № 4 VOL. I (JuLIO 2021) PÁGs. 129-160. ISSN: 2735-6523 
Rodríguez, M. (2010). La invisibilidad estadística étnico-racial negra, afrocolombiana, raizal y palenquera en Colombia. Trabajo Social (12), 89-99.

Romero, A. (2010). Educación por y para indígenas y afrocolombianos: las tecnologías de la etnoeducación. Revista Internacional de Investigación en Educación, 3 (5),167-182, III(5), 167-182.

Silva, J. (2010). Doutoras professoras negras: o que nos dizem os indicadores oficiais. Perspectiva, 28(1), 19-36.

Soler, S. (2009). La escuela y sus discursos. Los textos escolares como instrumentos de exclusión y segregación. Sociedad y Discurso (15), 107-124.

Stake, R. (1999). Investigación con estudio de casos. Madrid: Ediciones Morata.

UNESCO. (2012). Atlas mundial de la igualdad de género en la educación. París: Ediciones Unesco.

Vásquez-Padilla, D. (2019). ¿Somos conscientes del racismo? Cómo las categorías étnico-raciales, el color de la piel y el mestizaje inciden en el reconocimiento del racismo en Colombia. Sociedad y Economía, 36, 8-30. https://doi.org/10.25100/sye.v0i36.5932

Viveros, M. (s.f.). Equidad e inclusión en la educación superior. Algunos aportes a la discusión desde la Escuela de Estudios de Género de la Universidad Nacional de Colombia. http://www.bdigital.unal.edu.co/7409/1/maraviversovigoya1.pdf

Viveros, M. y Gil, F. (2010). Género y generaciones en las experiencias de ascenso social de personas negras en Bogotá. Maguaré, 24, 99-130.

Vizcarra, I. y Vélez, G. (2007). Género y éxito científico en la Universidad Autónoma del Estado de México. Revista Estudios Feministas, 15(3), 581-608.

Wade, P. (1997a). Race and ethnicity in Latin América. Chicago: Pluto Press. 
(1997b). Gente negra, nación mestiza. Dinámicas de las identidades raciales en Colombia. Bogotá: Ediciones Uniandes.

------(2009). Defining Blackness in Colombia. Journal de la Société des Américanistes 95(1), 165-184.

Walby, S. (Abril de 2003). Modernities /globalization /complexities. Paper presented to conference of the British Sociological Association, University of York.

-------(2007). Complexity Theory, Systems Theory, and Multiple Intersecting Social Inequalities. Philosophy of the Social Sciences, 37 (4), 449-470. DOI: $\quad 10.1177 / 0048393107307663$ http://pos.sagepub.com (2009). Globalization and Inequalities: Complexity and Contested Modernities. London: Sage.

(2011). El futuro del feminismo. Cambridge, Reino Unido Malden, Massachusetts: Polity Press.

Walby, S. y Allen, J. (2004). Violencia doméstica, agresión sexual y acecho: Hallazgos de la British Crime Survey (PDF). Londres: Dirección de Desarrollo y Estadísticas, Home Office Research. Documento de estudio de investigación del Ministerio del Interior, No. 276, basado en una consultoría con la British Crime Survey del Ministerio del Interior.

Weber, M. (1997). Economía y sociedad. Bogotá: Fondo de Cultura Económica. Weldon, L. (2006). The Structure of Intersectionality: A comparative Politics of Gender. Politics and Gender, 2(2), 235-248.

Wright, E. (1989). Reflexionando, una vez más, sobre el concepto de estructura de clases en The Debate on Classes (comp.). Londres: Verso. Yuval-Davis, N. (2006). Intersectionality and Feminist Politics. European Journal of Women's, 13(3), 193-209. 